\title{
Dependence of Curie temperature on the thickness of an ultrathin ferroelectric film
}

\author{
Emad Almahmoud, ${ }^{1}$ Igor Kornev, ${ }^{2}$ and L. Bellaiche ${ }^{3}$ \\ ${ }^{1}$ Physics Department, Umm Al-Qura University, Makkah 21421, KSA \\ ${ }^{2}$ Laboratoire SPMS, UMR 8580 du CNRS, Ecole Centrale Paris, 92295 Chatenay-Malabry, France \\ ${ }^{3}$ Physics Department, University of Arkansas, Fayetteville, Arkansas 72701, USA
}

(Received 6 December 2009; published 12 February 2010)

\begin{abstract}
The thickness dependency of the Curie temperature in stress-free $\mathrm{Pb}\left(\mathrm{Zr}_{0.5} \mathrm{Ti}_{0.5}\right) \mathrm{O}_{3}$ ultrathin films under open-circuit conditions is revealed from the computation of some nontrivial statistical quantities (such as fourth-order cumulants), via a first-principles-based technique. For thicknesses above $16 \AA$, this dependency follows the usual finite-size scaling law with a critical exponent that is consistent with the one associated with the three-dimensional-random-Ising universality class. On the other hand, the Curie temperature-versus-film's thickness curve deviates from this scaling law below $12 \AA$ while being rather well described by an empirical equation down to $8 \AA$.
\end{abstract}

DOI: 10.1103/PhysRevB.81.064105

PACS number(s): 77.80.B-, 64.60.F-, 68.65.-k, 77.55.-g

\section{INTRODUCTION}

Finite-size effects in dipolar (magnetic or ferroelectric) ultrathin films is a topic of growing interest for both technological and fundamental reasons-see, e.g., Refs. 1-8, and references therein. One particularly important issue in these low-dimensional systems is the dependency of their Curie temperature (that is, the highest temperature at which a spontaneous magnetization/polarization exists) on the film's thickness. The technological relevance of knowing such dependency partly stems from the possibility of designing improved devices since many properties fundamentally depend on the Curie temperature. Finite-size scaling theory predicts that this critical temperature shifts to lower temperatures than that of the bulk when one or more of the material's dimension(s) is reduced to an atomic size, ${ }^{9}$ with the shift being given by ${ }^{10}$

$$
\delta T=\left[T_{c}(\infty)-T_{c}(d)\right] / T_{c}(\infty)=\left(\frac{\xi_{0}}{d}\right)^{\lambda}
$$

where $T_{c}(\infty)$ and $T_{c}(d)$ are the Curie temperatures of the bulk material and of the film having a thickness denoted by $d$, respectively. $\xi_{0}$ and $\lambda$ are physically meaningful parameters for the corresponding bulk system: $\xi_{0}$ is its correlation length (interpolated down to $T=0 \mathrm{~K}$ ) while $\lambda$ is a critical exponent characterizing the so-called universality class to which this system belongs to (examples of universality class are threedimensional (3D) Heisenberg, two-dimensional Ising, etc.). However, a deviation of the $T_{c}(d)$-versus- $d$ curve from Eq. (1) was experimentally found in Refs. 11-13 for magnetic films below a critical thickness (note that such deviation carries important information, such as, e.g., the possible predominant role of surface on the transition temperature of ultrathin films). As a result, it was recently advocated ${ }^{14-16}$ that the behavior of the Curie temperature versus thickness can be fitted for a wider thickness range by considering $T_{c}(d)$ rather than $T_{c}(\infty)$ in the denominator of the relative shift in Curie temperature, that is by using

$$
\Delta T=\left[T_{c}(\infty)-T_{c}(d)\right] / T_{c}(d)=\left(\frac{\xi_{0}^{\prime}}{d}\right)^{\lambda^{\prime}}
$$

where $\lambda^{\prime}$ and $\xi_{0}^{\prime}$ do not possess any clear physical meaning, ${ }^{12,14}$ unlike $\lambda$ and $\xi_{0}$. Interestingly, the thickness dependency of the Curie temperature has been observed and tested against Eqs. (1) and (2) for a variety of lowdimensional magnetic systems ${ }^{11,14,17}$ but never in ferroelectrics, to the best of our knowledge. In other words, crucial characteristics of an important class of materials are currently unknown. In particular, one may wonder which relation among Eqs. (1) and (2) (if any) better describes the transition temperatures of ferroelectric thin films. In case that Eq. (1) holds in ferroelectric films (for any thickness or "only" above some critical thickness), one may also wonder if the involved critical exponent, $\lambda$, is consistent with a given universality class since there has been an intense debate for many years whether ferroelectrics belong to some universality classes or not (see, e.g., Ref. 18, and references therein). This paucity of knowledge may arise from the fact that many effects can affect the intrinsic $T_{c}(d)$-versus- $d$ curve in ferroelectric thin films. Examples of such effects are the increasing importance of depolarizing fields when decreasing the film's thickness ${ }^{19}$ or the increasing release of the strain arising from the substrate for thicker films. ${ }^{20}$ First-principlesbased approaches may thus be of great help to address the issues mentioned above since these methods have the flexibility to impose identical mechanical and/or electrical boundary conditions for ferroelectric films of different thicknesses-in addition to be accurate. However, predicting transition temperatures with these schemes is by no means trivial, and even when succeeding to do so, one has to face the undesirable fact that the computed critical temperatures of a given periodic system practically depend on the size of the supercell used to mimic such system. ${ }^{21,22}$

In this paper, we combine first-principles-derived approaches with statistical (e.g., Binder-cumulant ${ }^{23}$ ) techniques to determine the thickness dependency of the Curie temperature in $\mathrm{Pb}\left(\mathrm{Zr}_{0.5} \mathrm{Ti}_{0.5}\right) \mathrm{O}_{3}(\mathrm{PZT})$ thin films that are under stressfree and open-circuit electrical boundary conditions. As we 
will see, such determination provides the answer to the questions stated above. In particular, we numerically found that (i) Eq. (1) holds for thicknesses above $16 \AA$ with a $\lambda$ parameter being consistent with the corresponding critical exponent of the 3D-random-Ising universality class; and (ii) there is a breakdown of Eq. (1) for thicknesses below $12 \AA$ while Eq. (2) fits rather well the transition temperatures of the films for any thickness down to $d=8 \AA$.

The paper is organized as follows. Section II describes the first-principles-based approach used in this work. Section III reports and discusses our predictions while Sec. IV provides some conclusions.

\section{METHOD}

Here, we investigate (001) disordered $\mathrm{Pb}\left(\mathrm{Zr}_{0.5} \mathrm{Ti}_{0.5}\right) \mathrm{O}_{3}$ thin films that are $\mathrm{PbO}$ terminated. Such films are mimicked by $L \times L \times m$ supercells that are periodic along the $x$ and $y$ axes while finite along the $z$ axis-where the $x, y$, and $z$ axes are along the pseudocubic [100], [010], and [001] directions, respectively. The number of $B$ sites in each (001) layer of the supercell is thus $L \times L$ while $m$ is the number of (001) $B$-site layers (the thickness of the film is thus around $d=4 m$, when expressed in $\AA$ ). For computational reasons, we will limit ourselves to $m$ varying between 1 and 12 . The total energy $E_{t o t}$ of such low-dimensional system is written as ${ }^{24}$

$$
\begin{aligned}
E_{\text {tot }}\left(\{u(i)\},\{v(i)\}, \eta,\left\{\sigma_{i}\right\}\right)= & E_{\text {mat }}\left(\{u(i)\},\{v(i)\}, \eta,\left\{\sigma_{i}\right\}\right) \\
& +P \sum_{j} u_{z}(j)+T \sum_{j} v_{z}(j),
\end{aligned}
$$

where $\mathbf{u}(i)$ is the (B-centered) local soft mode in the unit cell $i$ of the film and is directly proportional to the electrical dipole at that site. $\mathbf{v}(i)$ is the inhomogeneous strain around the $i$ site while $\eta$ is the homogeneous strain tensor. ${ }^{25}$ The local modes and both strains are allowed to fully relax in order to mimic a stress-free film. $\left\{\sigma_{i}\right\}$ characterizes the alloy configuration $^{26}$ - which is chosen to provide a realization of a disordered system and is frozen in our simulations. $E_{\text {mat }}$ represents the intrinsic ferroelectric and elastic interactions inside the film. Its analytical expression and first-principlesderived parameters are those given in Ref. 26 for PZT bulk, except for the dipole-dipole interactions for which we use the analytical expressions derived in Refs. 27 and 28 for films under ideal open-circuit electrical boundary conditions. $E_{\text {mat }}$ includes terms describing on-site local-mode self-energy, short-range, and dipole-dipole interactions between local modes, elastic energy, the coupling between local modes and elastic variables, and alloying-related terms. Moreover, the $P$ and $T$ parameters appearing in Eq. (3) quantify how the existence of the (free) upper surface affects the out-of-plane components $\left(u_{z}\right.$ and $\left.v_{z}\right)$ of the local modes and inhomogeneous strains, respectively, at the $j$ sites closest to this upper surface [such parameters are determined from first-principles calculations on a PbO-terminated (001) 17-atom slab $\left.{ }^{24}\right]$. Furthermore, no term analogous to the last two expressions of Eq. (3) is considered at the lower surface since this latter characterizes the substrate/film interface and that we consider the substrate to be inert. Technically, we use the total

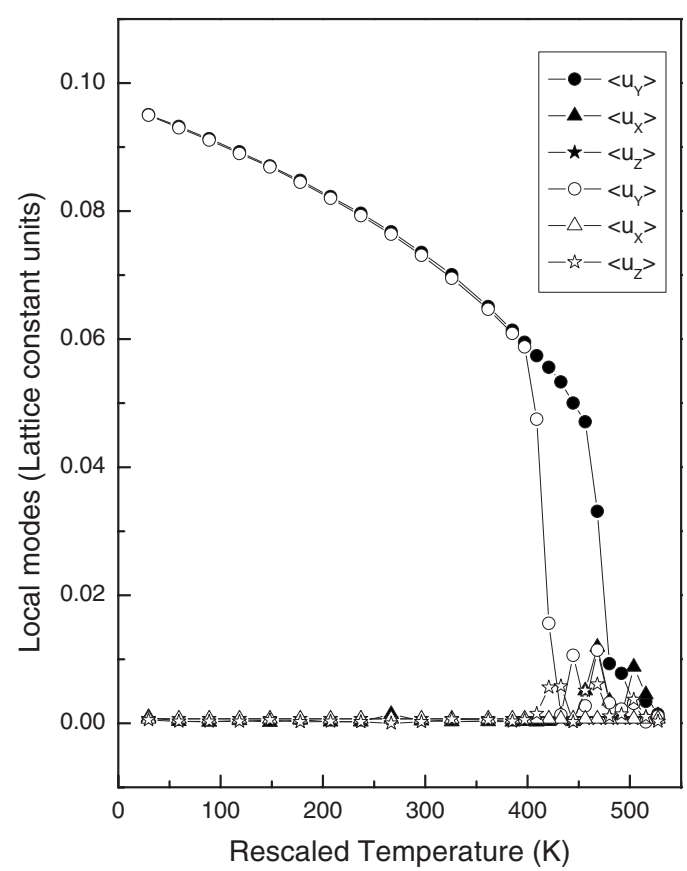

FIG. 1. Cartesian components of the supercell average of the local modes in a 20 - $\AA$-thick stress-free $\mathrm{Pb}\left(\mathrm{Zr}_{0.5} \mathrm{Ti}_{0.5}\right) \mathrm{O}_{3}$ film under open-circuit electrical boundary conditions, as a function of temperature. The open symbols refer to simulations performed with a $16 \times 16 \times 5$ supercell while the filled symbols display the results for a $26 \times 26 \times 5$ supercell. The temperature has been rescaled to match the experimental Curie temperature of PZT bulk.

energy of Eq. (3) in Monte Carlo (MC) simulationsperformed with the Metropolis algorithm ${ }^{29}$ — and use a huge number of Monte Carlo sweeps (up to 20 million) for each temperature to reach convergency. The local soft modes and strains are collected at each Monte Carlo sweep, and the temperature is practically decreased by small steps.

\section{RESULTS}

Figure 1 displays the predicted temperature behavior of the Cartesian coordinates $\left(\left\langle u_{x}\right\rangle,\left\langle u_{y}\right\rangle\right.$, and $\left.\left\langle u_{z}\right\rangle\right)$ of the localmode vectors averaged over the supercell sites and over the Monte Carlo sweeps, in the 20 - $\AA$-thick $(m=5)$ film. ${ }^{30}$ Results for two different supercell sizes (namely, associated with $L$ $=16$ or $L=26$ ) are shown there. For both sizes, each coordinate is close to zero at high temperature, indicating a paraelectric phase. As the temperature is decreased and passes through a critical value, $\left\langle u_{y}\right\rangle$ rapidly increases when the temperature decreases while $\left\langle u_{x}\right\rangle$ and $\left\langle u_{z}\right\rangle$ remain nearly null-as consistent with Ref. 24. This indicates the formation of a ferroelectric phase (via a second-order transition) with a polarization lying along an in-plane $\langle 010\rangle$ direction, below this critical temperature. Such in-plane direction leads to a vanishing depolarizing field. Interestingly, Fig. 1 also shows that the computed critical temperature of PZT thin films having a given thickness strongly depends on the $L$ lateral supercell length, and increases as $L$ increases. Such size dependencies were previously reported for ferromagnetic thin films. ${ }^{31,32}$ They also indicate that determining the "real" $\mathrm{Cu}$ - 


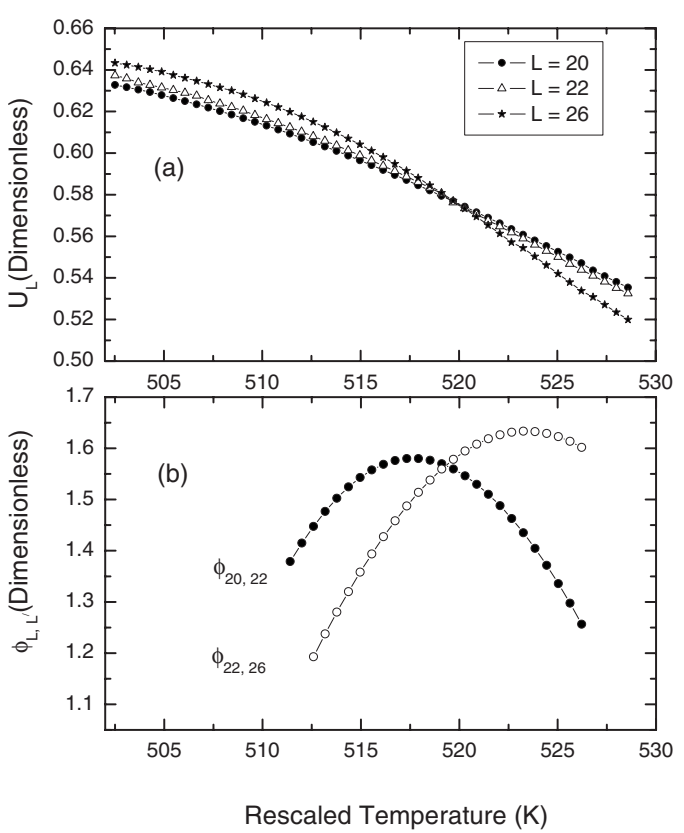

FIG. 2. Temperature dependency of fourth-order cumulants [part (a)] and of $\phi_{L, L^{\prime}}(T)$ functions [part (b)], for different lengths of the periodic supercells, in a 20 - $\AA$-thick stress-free $\mathrm{Pb}\left(\mathrm{Zr}_{0.5} \mathrm{Ti}_{0.5}\right) \mathrm{O}_{3}$ film under open-circuit electrical boundary conditions. The temperature has been rescaled to match the experimental Curie temperature of PZT bulk.

rie temperature, $T_{c}(d)$, associated with a specific thickness $d$ (i.e., the critical temperature corresponding to the case of $L$ $\rightarrow \infty$ ) is practically impossible when solely using the simulations of a fixed periodic supercell. In fact, as we will see below, this determination can be accomplished by combining predictions offered by our numerical tool with particularly relevant statistical features. More precisely, one can compute the fourth-order cumulant $U_{L}{ }^{32}$ defined as

$$
U_{L}=1-\frac{\left\langle u^{4}\right\rangle}{3\left\langle u^{2}\right\rangle^{2}},
$$

where " \langle\rangle " denotes the average over the MC sweeps, and where $u^{2}$ and $u^{4}$ represent the square and fourth power of the magnitude of the local modes (averaged over the $L \times L \times m$ supercell sites for each MC sweep), respectively. Such latter quantities are provided by our computational scheme for each investigated temperature. Note that $U_{L}$ is practically calculated by taking advantage of the so-called reweighting technique. ${ }^{33}$ Interestingly, it is well established that $U_{L}$ should be independent of $L$ for a single temperature, that is $T_{c}(d) .{ }^{32}$ Such important fact is indeed numerically confirmed in Fig. 2(a), that shows the temperature dependency of the fourth-order cumulant of a PZT thin film with $m=5$ for three different lateral supercell sizes (namely, $L=20,22$, and 26). One can indeed clearly see that these three different curves all intersect at a temperature equal to $520 \mathrm{~K}$, which is thus taken to be the real Curie temperature of the 20- $\AA$-thick PZT film.

To further confirm this value, we also computed the function defined as

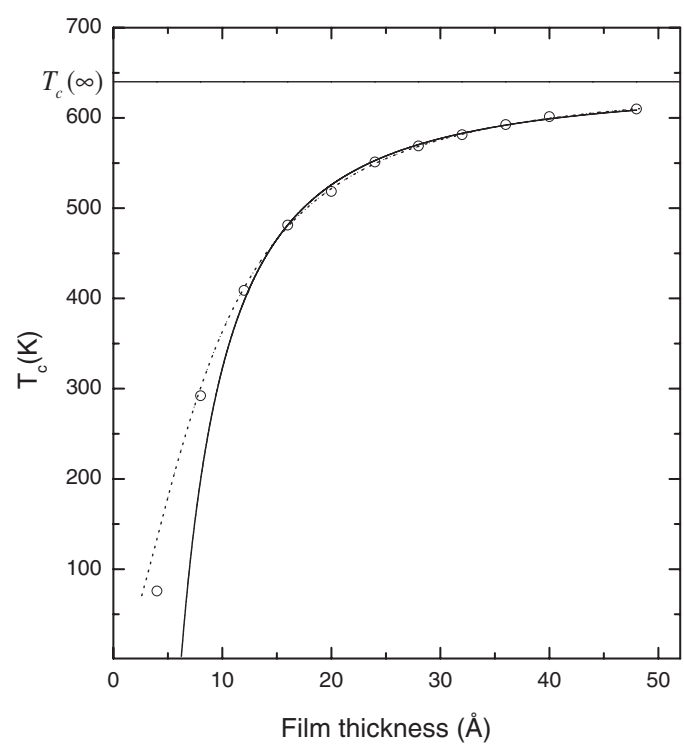

FIG. 3. Thickness dependency of the Curie temperature in stress-free $\mathrm{Pb}\left(\mathrm{Zr}_{0.5} \mathrm{Ti}_{0.5}\right) \mathrm{O}_{3}$ films under open-circuit electrical boundary conditions. The solid line shows the fit of the numerical data to Eq. (1) while the dashed line represents the fit of the same data but to Eq. (2). The uncertainties of the numerical data are smaller than the size of the dot used to display such data and are thus not shown for clarity. The horizontal bold line shows the Curie temperature of PZT bulk. The temperature has been rescaled to match the experimental Curie temperature of PZT bulk.

$$
\phi_{L, L^{\prime}}(T) \equiv \frac{\ln \left[\chi\left(L^{\prime}, T\right) / \chi(L, T)\right]}{\ln \left(L^{\prime} / L\right)},
$$

where

$$
\chi(L, T)=\frac{\left(L^{2} d\right)\left[\left\langle u^{2}\right\rangle-\langle|u|\rangle^{2}\right]}{T},
$$

where $T$ denotes the temperature, and where the statistical averages of the $u$-dependent terms are once again determined by our presently used numerical scheme for $L \times L \times m$ supercells. Reference 23 indicates that the $T_{c}(d)$ Curie temperature should be the temperature at which the $\phi_{L, L^{\prime}}(T)$ and $\phi_{L^{\prime}, L^{\prime \prime}}(T)$ functions intersect for any two pairs of sizes, $\left(L, L^{\prime}\right)$ and $\left(L^{\prime}, L^{\prime \prime}\right)$ with $L^{\prime \prime}>L^{\prime}>L$. Figure 2(b) indeed shows that such intersection occurs between $\phi_{20,22}(T)$ and $\phi_{22,26}(T)$ for the PZT thin film having $m=5$. More precisely, this intersection happens at $519 \mathrm{~K}$, which is in very good agreement with the Curie temperature previously obtained using the fourth-order cumulant, see Fig. 2(a). The (tiny) difference between the $T_{c}(d)$ obtained when using the crossing of the $U_{L}$ 's or of the $\phi_{L, L^{\prime}}(T)$ 's practically provides our uncertainty for the Curie temperature of the investigated film. Moreover, the temperature crossings of the fourth-order cumulants and of the $\phi_{L, L^{\prime}}(T)$ functions were numerically found to occur for all the considered thicknesses. We thus are in position to reveal the thickness dependency of the real Curie temperature in PZT thin films, as displayed in Fig. 3. Furthermore, the resulting data of this latter figure are fitted using the wellestablished Eq. (1), as well as the empirical law described by 
Eq. (2). ${ }^{34}$ Several striking features can be clearly seen. First of all, there is no critical thickness below which ferroelectricity vanishes in stress-free PZT film under open-circuit conditions, as consistent with Refs. 24 and 35. As a matter of fact, Fig. 3 indicates that the Curie temperature is equal to $76 \mathrm{~K}$ for the thinnest possible system (i.e., for $m=1)$. Second, $T_{c}(d)$ decreases as the film becomes thinner, as previously found for magnetic and ferroelectric nanostructures. ${ }^{2,11,12,14,31,36}$ Third, such decrease is very well described by the usual finite-size scaling relationship of Eq. (1) but only for thickness above or equal to $16 \AA$. More precisely, $T_{c}(d)$ deviates more and more from the fit to Eq. (1) as the thickness decreases below $12 \AA$. Such kind of deviation has also been recently found in magnetic ultrathin films, ${ }^{11-13,37}$ and arises from the increasingly important role of surface effects relative to bulk effects when decreasing the film's thickness. Interestingly, the two degrees of freedom of Eq. (1) are numerically found to be $\xi_{0}=6.21 \pm 0.20 \AA$, and $\lambda=1.47 \pm 0.03$. The former parameter indicates a correlation length corresponding to the third-nearest-neighbors' distance (in the $B$ sublattice) for PZT bulks. The latter parameter confirms the prediction of Ref. 18, namely, that the critical exponents of $\mathrm{Pb}\left(\mathrm{Zr}_{0.5} \mathrm{Ti}_{0.5}\right) \mathrm{O}_{3}$ bulks are consistent with those of the 3D-random-Ising universality class-for which $\lambda$ has been reported to be around $1.46 .{ }^{38}$ Finally, Fig. 3 demonstrates that the empirical relationship of Eq. (2) can nicely reproduce the Curie temperatures down to a thickness of $8 \AA$, with $\lambda^{\prime}=1.75 \pm 0.03$ and $\xi_{0}^{\prime}=8.55 \pm 0.20 \AA$. In other words, Eq. (2) somehow captures the effect of surface on the transition temperatures in ultrathin systems, as also found in low-dimensional magnets. ${ }^{12,14}$ However, the loss of a fundamental feature occurs with such capture, namely, the result- ing $\lambda^{\prime}$ [which is always larger than $\lambda$ (Refs. 12 and 39)] is not associated with an universality class.

Note that we have also tested the effect of the $P$ and $T$ parameters of Eq. (3) on the critical temperature, by turning off both of them in the simulations, and found that $T_{c}(d)$ is not affected by these parameters for any investigated thickness. $^{40}$

\section{CONCLUSIONS}

In summary, we have revealed the thickness dependency of the Curie temperature in $\mathrm{Pb}\left(\mathrm{Zr}_{0.5} \mathrm{Ti}_{0.5}\right) \mathrm{O}_{3}$ ultrathin films, by computing nontrivial statistical quantities (such as fourthorder cumulants) from first-principles-based schemes. It is found that the empirical relation of Eq. (2) reproduces rather well such dependency for thickness down to $8 \AA$, in contrast with the usual finite-size scaling law of Eq. (1) that breaks down below $16 \AA$. The resulting value for the parameter $\lambda$ appearing in Eq. (1) (for thicknesses above $16 \AA$ ) also further confirms that the critical exponents of $\mathrm{Pb}\left(\mathrm{Zr}_{0.5} \mathrm{Ti}_{0.5}\right) \mathrm{O}_{3}$ bulks are consistent with the 3D-random-Ising universality class, as recently predicted. ${ }^{18}$ We are confident that our present work provides a deeper knowledge of nanoscience, phase transitions, and critical behaviors in dipolar systems.

\section{ACKNOWLEDGMENTS}

We acknowledge support from ONR under Grants No. N00014-04-1-0413 and No. N00014-08-1-0915, NSF under Grants No. DMR-0080054 (C-SPIN), No. DMR-0701558, and No. DMR-0404335, and DOE under Grant No. DESC0002220.
${ }^{1}$ C. H. Ahn, K. M. Rabe, and J. M. Triscone, Science 303, 488 (2004).

${ }^{2}$ D. D. Fong, G. B. Stephenson, S. K. Streiffer, J. A. Eastman, O. Auciello, P. H. Fuoss, and C. Thompson, Science 304, 1650 (2004).

${ }^{3}$ J. F. Scott and C. A. Paz de Araujo, Science 246, 1400 (1989).

${ }^{4}$ M. Dawber, K. M. Rabe, and J. F. Scott, Rev. Mod. Phys. 77, 1083 (2005).

${ }^{5}$ I. A. Kornev, H. Fu, and L. Bellaiche, J. Mater. Sci. 41, 137 (2006).

${ }^{6}$ M. T. Johnson, P. J. H. Bloemen, F. J. A. den Broeder, and J. J. de Vries, Rep. Prog. Phys. 59, 1409 (1996).

${ }^{7}$ P. Poulopoulos and K. Baberschke, J. Phys.: Condens. Matter 11, 9495 (1999).

${ }^{8} \mathrm{~K}$. Binder, in Phase Transitions and Critical Phenomena, edited by C. Domb and M. S. Green (Academic, New York, 1983), Vol. 8.

${ }^{9}$ M. E. Fisher and M. N. Barber, Phys. Rev. Lett. 28, 1516 (1972).

${ }^{10}$ G. A. T. Allan, Phys. Rev. B 1, 352 (1970).

${ }^{11}$ Y. Li and K. Baberschke, Phys. Rev. Lett. 68, 1208 (1992).

${ }^{12}$ T. Ambrose and C. L. Chien, J. Appl. Phys. 79, 5920 (1996).

${ }^{13}$ R. Zhang and R. F. Willis, Phys. Rev. Lett. 86, 2665 (2001).
${ }^{14}$ F. Huang, G. J. Mankey, M. T. Kief, and R. F. Willis, J. Appl. Phys. 73, 6760 (1993).

${ }^{15}$ K. Binder and P. C. Hohenberg, Phys. Rev. B 9, 2194 (1974).

${ }^{16}$ J. R. Childress, C. L. Chien, and A. F. Jankowski, Phys. Rev. B 45, 2855 (1992).

${ }^{17}$ D. Lederman, C. A. Ramos, and V. Jaccarino, Phys. Rev. B 48, 8365 (1993).

${ }^{18}$ E. Almahmoud, I. Kornev, and L. Bellaiche, Phys. Rev. Lett. 102, 105701 (2009).

${ }^{19}$ J. Junquera and Ph. Ghosez, Nature (London) 422, 506 (2003).

${ }^{20}$ C. L. Canedy, H. Li, S. P. Alpay, L. Salamanca-Riba, A. L. Roytburd, and R. Ramesh, Appl. Phys. Lett. 77, 1695 (2000).

${ }^{21}$ K. Chen, A. M. Ferrenberg, and D. P. Landau, Phys. Rev. B 48, 3249 (1993).

${ }^{22}$ A. M. Ferrenberg and D. P. Landau, Phys. Rev. B 44, 5081 (1991)

${ }^{23}$ K. Binder and D. W. Heermann, Monte Carlo Simulations in Statistical Physics: An Introduction (Springer-Verlag, New York, 1988).

${ }^{24}$ E. Almahmoud, Y. Navtsenya, I. Kornev, H. X. Fu, and L. Bellaiche, Phys. Rev. B 70, 220102 (2004).

${ }^{25}$ W. Zhong, D. Vanderbilt, and K. M. Rabe, Phys. Rev. B 52, 6301 (1995). 
${ }^{26}$ L. Bellaiche, A. Garcia, and D. Vanderbilt, Phys. Rev. Lett. 84, 5427 (2000); Ferroelectrics 266, 41 (2002).

${ }^{27}$ I. Ponomareva, I. Naumov, I. Kornev, H. Fu, and L. Bellaiche, Phys. Rev. B 72, 140102 (2005).

${ }^{28}$ I. Naumov, arXiv:cond-mat/0505497 (unpublished).

${ }^{29}$ N. Metropolis, A. Rosenbluth, M. Rosenbluth, M. Teller, and E. Teller, J. Chem. Phys. 21, 1087 (1953).

${ }^{30}$ Here, we rescaled our theoretical temperature by multiplying it by a constant coefficient, in order to match the experimental Curie temperature of PZT bulk. Such rescaling has been found to accurately reproduce various experimental temperaturedependent data (Ref. 26), and has no effect on the investigated parameters of Eqs. (1) and (2) — as consistent with the fact that these two equations involve a ratio between different temperatures.

${ }^{31}$ Y. Laosiritaworn, J. Poulter, and J. B. Staunton, Phys. Rev. B 70, 104413 (2004).

${ }^{32}$ K. Binder, Rep. Prog. Phys. 50, 783 (1987).

${ }^{33}$ A. M. Ferrenberg and R. H. Swendsen, Phys. Rev. Lett. 61, 2635 (1988).

${ }^{34}$ These fits were practically done by only considering our numerical data for $m$ greater than, or equal to, 7 , for two main reasons: (1) to avoid including some possible surface effects that manifest themselves in the thinnest possible films [such effects are the ones that can lead to a deviation of the data with respect to
Eq. (1)]; and (2) because the parameters of both Eqs. (1) and (2) are found to be converged (i.e., nearly independent of the points included in the fit) when considering the data above $m=5$.

${ }^{35}$ Z. Wu, N. Huang, Z. Liu, J. Wu, W. Duan, B.-L. Gu, and X.-W. Zhang, Phys. Rev. B 70, 104108 (2004).

${ }^{36}$ J. E. Spanier, A. M. Kolpak, J. J. Urban, I. Grinberg, L. Quyang, W. S. Yun, A. M. Rappe, and H. Park, Nano Lett. 6, 735 (2006).

${ }^{37}$ E. Weschke, H. Ott, E. Schierle, C. Schossler-Langeheine, D. V. Vyalikh, G. Kaindl, V. Leiner, M. Ay, T. Schmitte, H. Zabel, and P. J. Jensen, Phys. Rev. Lett. 93, 157204 (2004).

${ }^{38}$ P. H. R. Barbosa, E. P. Raposo, and M. D. Coutinho-Filho, Phys. Rev. B 72, 092401 (2005); M. Hasenbusch, F. Parisen Toldin, A. Pelissetto and E. Vicari, J. Stat. Mech.: Theory Exp. (2007) P02016.

${ }^{39}$ M. Henkel, S. Andrie, P. Bauer, and M. Piecuch, Phys. Rev. Lett. 80, 4783 (1998).

${ }^{40}$ The insensitivity of the Curie temperature on the $P$ and $T$ parameters of Eq. (3) is due to the fact that the polarization lies in plane for temperature below the critical temperature while these two parameters characterize effects associated with the out-ofplane direction. On the other hand, adding an energetic term characterizing the surface-induced change in in-plane dipoles in Eq. (3) can strongly affect the Curie temperature by, e.g., making it increase as the film thickness decreases. 